\title{
Real-Time Noise Classification of Medical Image via Online Machine Learning Algorithm
}

\author{
${ }^{1}$ Yuchou Chang \\ ${ }^{1}$ Computer Science and Engineering Technology Department, University of Houston - Downtown, \\ Houston, United States \\ changy@uhd.edu
}

\begin{abstract}
Medical image is generally deteriorated by noise due to signal acquisition, signal processing, and other reasons. Noise classification of medical image is able to enhance post-processing tasks like medical image segmentation, registration, and analysis. Due to real-time requirements of medical image analysis for clinical applications, noise classification of medical image is desired to be fast for meeting real-time requirements. On the other hand, online learning algorithms have been studied for processing online data in real-time mode, which can produce rapid learning model based on adjustments of new incoming data. In this paper, we investigate perceptron algorithm - a classical online learning method for noise classification in parallel magnetic resonance imaging (pMRI). Noise generated in $\mathrm{pMRI}$ is quickly classified and online classification model is updated in real-time simultaneously. Experimental results demonstrate that noise and brain tissues existing MR images is able to be classified dynamically with the perceptron algorithm.
\end{abstract}

Keywords: Noise Classification, Online Learning Algorithm, Medical Image, Noise Distribution, and Parallel Magnetic Resonance Imaging.

\section{Introduction}

Image acquisition and processing is generally accompanied by noise, which is generated in the final image with various reasons during imaging stages. Noise can deteriorate image quality. There are two types of causes that generate noise: external causes and internal causes. Each type of noise is composed of several sources of noise. For example, external noise refers to the noise caused by the external interference of the system by the electromagnetic wave or the power source entering the system. This type of noise may be Gaussian noise, impulse noise and other noise synthesis cumulative. Identification and classification of medical image noise level is important for other tasks like image denoising [1-3], object recognition [4-6], and motion tracking [7-9]. On the other hand, real-time is another requirement in many image-based applications like medical image guided intervention or surgery [10]. In the field of medical image acquisition and processing, due to imperfection of imaging system, transmission media and recording equipment, medical images are often contaminated by various noises during the formation and transmission of medical images. Noise is not related to the object of study as useless information, which disturbs the observability of the image. Therefore, the classification and identification of noise is important in medical image processing. If the image noise is not suppressed, it will be difficult to process the image directly, which may even lead physicians to make incorrect judgments when patient's condition is diagnosed. 
Yuchou Chang; Real-Time Noise Classification of Medical Image via Online Machine Learning Algorithm. Advances in I mage and Video Processing, Volume 5 No 6, December (2017); pp: 1-9

Online learning $[11,12]$ is a commonly used machine learning algorithm in the industry and can work well in many scenarios. Online Learning can make real-time and rapid learning model adjustments based on online feedback data so that the learning model can reflect online changes timely and improve the accuracy of online prediction. The process of online learning includes presenting the learning model's predictions to the user, collecting user feedback data, and then training the learning model to form a closed-loop system. The ideal algorithm is to be able to get a good learning model with a small amount of data. Online learning uses only current and past data and the future is unknown. Therefore, for online learning, it pursues the best strategy that can be designed with all the data dynamically. The difference with this optimal strategy is called regret: regret doesn't choose the optimal strategy rightly from the beginning. With more data over time, the difference will become smaller. Because any assumptions about the data is not made, the optimal strategy may be not perfect (for example, classifying all data correctly).

In magnetic resonance imaging (MRI), the technique of sensitivity encoding (SENSE) [13] uses a phased array coil with different sensitivities to place the target around. In order to maintain the spatial resolution unchanged, sampling rate in $k$-space is decreased to reduce the number of phase-encoding sampling steps, so that the imaging speed is accelerated. As a parallel MRI technique, SENSE requires to use the coil sensitivity profile unfolding aliasing image, and the coil sensitivity map is pre-scanned. Parallel MRI techniques lead to a significant reduction in signal-to-noise ratio. For example, if reduction factor $\mathrm{R}=2$, the image SNR decreases by $\sqrt{2}$, which is around $40 \%$. This disadvantage is usually not only a problem in high fields (where higher signal-to-noise ratios are higher) and but also in certain anatomical areas (e.g. relatively symmetrical and uniform brains). Such reconstruction errors increase as the acceleration factor $(R)$ increases.

In order to characterize noise distribution in SENSE reconstruction based MR image, an online learning framework is proposed in the paper. Online learning is applied to classify and identify noise distribution in real-time mode of reconstructed MR images. The paper is organized as follows. The first section introduces context of the proposed research work. The second part presents the background of the relevant methods. The third one gives the proposed method of the real-time noise classification of MR images. Experimental results and conclusion will be provided in the fourth and fifth sections, respectively.

\section{Background}

In traditional machine learning method, after the learning model is trained, the update cycle of the learning model will be longer since training dataset cannot be updated quickly. Therefore, after the model is online, it is generally static (it will not change for a period of time). Any interaction with the online learning model (assuming if the prediction is wrong) can only be corrected at the next stage of the model update. Online learning algorithms are able to adjust the learning model dynamically based on the results of online prediction. If wrong prediction or classification is produced by the learning model, it will be corrected in time. Therefore, online learning can reflect online changes in a real-time manner. Online Learning can make real-time and rapid learning model adjustments based on online feedback data, so that the learning model can timely reflect online changes and improve the accuracy of online prediction. The optimization goal of Online Learning is to minimize the total loss function.

As shown in the Figure 1, a closed-loop system of online learning contains several steps. At time $t$, learning model receives data $x_{t}$, learning model provides a prediction or classification strategey $h_{t}$, and environment will give the correct answer $y_{t}$. Learning model will have a loss $l\left(h_{t}, y_{t}\right)$ at the moment. 


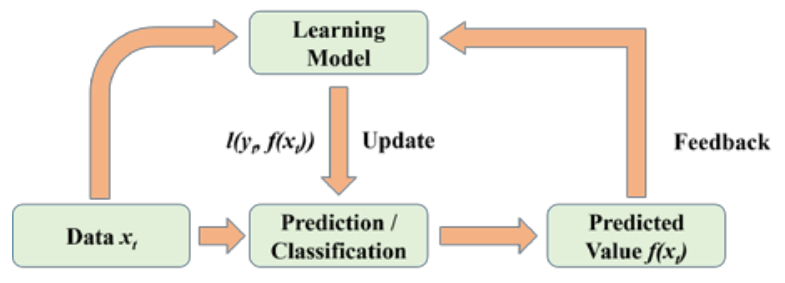

Figure 1. Framework of online learning algorithm.

Learning model learns domain knowledge when learning model receives data each time. The repeated steps of online learning is as follows: (1) receiving data $x_{t},(2)$ learning model selects a strategy $h_{t}$ and make a prediction $h_{t}\left(x_{t}\right)$, and (4) repeat the above steps to produce loss each time. In order to evaluate learning quality each time, the concept of regret is defined as

$$
R(T)=\sum_{t=1}^{T} l_{t}\left(h_{t}\right)-\min \sum_{t=1}^{T} l_{t}(h), h \in \mathcal{H}
$$

, where $h$ is the optimal strategy of learning model is to make a good $h_{t}=f\left(x_{t}\right)$ each time to make $R(T)$ minimum. The optimal strategy is to fix $h$, but if we can choose a good $h_{t}$ to adapt to the learning problem $\left(x_{t}, y_{t}\right)$ every time, the total loss may be smaller. But this is difficult. since a good strategy $h_{t}$ has to be set and correct answer $y_{t}$ should be known. If the learning problem is very different from the previous one and the answer is difficult to be obtained. For the optimal strategy, if all the learning problems and answers are known in advance, so optimal strategy is more likely to have a minimum total loss. Therefore, the average regretR $(T) / T$ is considered since it is smaller with $T$ becomes smaller. If an online algorithm is not no-regret, it means:

$$
\lim _{n \rightarrow \infty} \frac{R(T)}{T} \rightarrow 0
$$

On the other hand, a parallel MRI technique is used to apply online learning algorithm for identifying noise distribution. MR images can be generated by fast parallel MR imaging to speed up imaging. SENSE technology is one of the widely used parallel MR image reconstruction techniques. SENSE's rapid performance makes patients more comfortable and reduces clinical costs. Although more noise and aliasing artifacts are produced as a compromise of imaging acceleration in the SENSE method, it has been widely used in clinical applications. Acronyms [14] of SENSE implementation on five MR scanner vendors are shown in the Table 1 . The general SENSE equation is

$$
E f=d
$$

, where $d$ is a vector formed from k-space data on all channels, $f$ is the complete FOV image to be solved, and $\mathrm{E}$ is the sensitivity encoding matrix. Using an unwrapping operation, the signal is separated for each pixel in a reduced FOV to produce a unaliased, full field image. In addition, geometric factors, a priori signal-to-noise ratio (SNR) estimates, and important criteria for designing a coil array consist of

$$
g(\rho)=\sqrt{\left[\left(\mathrm{S}^{\mathrm{H}} \Psi^{-1} \mathrm{~S}\right)^{-1}\right]_{\rho, \rho}\left(\mathrm{S}^{\mathrm{H}} \Psi^{-1} \mathrm{~S}\right)_{\rho, \rho}}
$$

where $\rho$ is pixel, $\mathrm{S}$ is the sensitivity matrix, and $\Psi$ is the receiver noise covariance matrix. During parallel MR imaging, geometry related noise enhancement increases rapidly when reduction factor increases. The proposed method identifies the distribution of noise and aliasing artifacts by using geometric factors to identify enhanced edge detection of MR brain images. 
Table 1 shows acronyms of sensitivity encoding (SENSE) implementation on five MR scanner vendors.

\begin{tabular}{|c|c|}
\hline MR Scanner Vendors & $\begin{array}{c}\text { Acronyms of SENSE } \\
\text { Implementation }\end{array}$ \\
\hline Siemens & mSENSE \\
\hline General Electric (GE) & ASSET \\
\hline Philips & SENSE \\
\hline Hitachi & RAPID \\
\hline Toshiba & SPEEDER \\
\hline
\end{tabular}

\section{Proposed Method}

Perceptron $[15,16]$ is a supervised linear online classification algorithm. The algorithm divides the input examples into positive and negative ones. After learning the results, the algorithm estimates $w$ from the examples in the training data. The algorithm then predicts the category of input data based on the sign of the product of the feature vector $x$ and the parameter vector $w$. The perceptron algorithm accepts an input instance $x$ and determines its category based on the weight vector $w$. Let input $x$ be a d-dimensional real vector, $w$ is the weight vector of the model, and it is also a $d$ dimensional real vector. The binary perceptron divides the input $x$ into two categories according to the output function $w^{T} x$. It divides instance $x$ of $w^{T} x>0$ into positive ones. On the other hand, it divides $x$ into negative ones when $w^{T} x<0$. Given training data, what the perceptron algorithm learns is the model's weight vector $w$. Initialization weight vector $w$ is 0 . In iteratively determining the positive and negative cases of error, when positive examples are negative, $w(t+1) \leftarrow w(t)+x$, and when negative examples are positive, $w(t+1) \leftarrow w(t)-x$.

MRI noise [17] can worsen medical image analysis performance because many image analysis algorithms like segmentation and registration are sensitive to noise within the image. Noise also reduces the anatomy boundary accuracy in MR images. Noise generation in MR scans is complicated. It is related to electricity current, coil configuration, and magnetic fields. Noise should be suppressed in MR image. Suppose the input space (pixel values in MR image) is $X$ and the output space is $Y=\{-1$, +1 . Each feature vector $x \in X$ of the instance is corresponding to each point in the input space. The output $y \in Y$ represents the class (pixel's noise level) of the example. The function from input space to output space is

$$
p(x)=\operatorname{sign}(w \cdot x+b)
$$

, where $p$ is called perceptron, $w$ represent weight vector, and $b$ is bias. The $w \cdot x$ denotes the inner product of $w$ and $x$,

$$
\sum_{i=1}^{n} w_{i} x_{i}=w_{1} x_{1}+w_{2} x_{2}+\cdots+w_{n} x_{n}
$$

, where sign is sign function. It is

$$
p(x)=\left\{\begin{array}{rr}
+1, & \text { if } x>=0 \\
-1, & \text { else }
\end{array}\right.
$$

Sensitivity encoding (SENSE) is a typical image-based MRI reconstruction method. It is non-aliased in the image domain. After inverse Fourier transform of $k$-space data, SENSE reconstructs the aliased coil images. During the inverse Fourier transform, the field of view (FOV) is reduced by $1 / R$ ( $R$ is a reduction factor for undersampled $k$-space). The same information is contained in the smaller area, which leads to folded aliasing artifacts. According to the Eq. (1), the brain MR image is reconstructed by SENSE algorithm as shown in Figure 2 (d). It can be seen that the reconstructed image deteriorates due to noise and aliasing artifacts as a tradeoff of acceleration of the undersampled signal in $k$-space. 
In addition, the geometry factor aligning with noise distribution is generated as shown in Figure 2 (e). It can characterize the distribution of noise and aliasing artifacts appearing in the SENSE image in Figure 2 (d). The higher value of the geometric factor represents the lower SNR of the corresponding pixel location in the image. The geometric factor is able to guide the noise distribution and intensity in the reconstructed SENSE image. The proposed method uses geometric factor values to represent noise levels in the MR image domain. Noise and true pixel values should be classified into different classes for identifying noise. The value of $p(x)(+1$ or -1$)$ is used to classify whether MR image pixel $\mathrm{x}$ is true value $(+1)$ or noise $(-1)$. Perception machine is a linear classification model. All need to do is find an optimal $w$ and $b$ value that satisfies the separating hyperplane $w \cdot x+b=0$.

The purpose of the perceptron learning is to find a separation hyperplane that completely separates the true value pixels and noise instances of the training set. Since a pixel with higher geometry factor value represents higher level of noise, the neighbor pixels also have a high probability to be noisy pixels. For this reason, neighbor 8 pixels of the local $3 \times 3$ window are extracted and combined with the center pixel as a feature vector representing the local window noise level. To find such a hyperplane, the perceptron model parameters $w$ and $b$ are determined through minimizing the loss function. For the choice of loss function, distance from the misclassified point to the hyperplane is calculated as

$$
\frac{1}{\|w\|}\left|w \cdot x_{0}+b\right|
$$

, where $\|w\|$ is $L 2$ norm. For a misclassified pixel $\left(x_{i}, y_{i}\right)$, it has $-y_{i}(w \cdot x+b)>0$. The distance between this misclassified pixel and hyperplane is calculated as

$$
-\frac{1}{\|w\|} y_{i}\left(w \cdot x_{0}+b\right)
$$

Therefore, the loss function of all misclassified pixels to the hyperplane is defined as

$$
L(w, b)=-\sum_{x_{i} \in M} y_{i}\left(w \cdot x_{0}+b\right)
$$

, where $M$ is the set of all misclassified pixels in MR image. If there is no misclassified pixels, the loss function is zero. If there are fewer misclassified pixels and they are closer to the hyperplane, the loss function is also smaller. Perceptron learning algorithm is driven by misclassified pixels, so we use stochastic gradient descent method to alternatively solve the following minimization problem.

$$
\min _{w, b} L(w, b)=-\sum_{x_{i} \in M} y_{i}\left(w \cdot x_{0}+b\right)
$$

In this way, it can be expected through iteration that the loss function $L(w, b)$ value continues to decrease until it reaches zero.

\section{Experimental Results}

As shown in Figure 2 (a), the reference image 1 has little noise and aliasing artifacts. It is completely sampled but requires longer MR scanner acquisition time. Figure 2 (b) and (c) show a coil image and its coil sensitivity profile. Coil sensitivity creates noise and aliasing artifacts in the reconstructed MR image as shown in Figure 2 (d), thereby reducing image quality. It can be seen that noise exists in white matter, gray matter, and cerebrospinal fluid (CSF) of brain image. The corresponding geometric factor map as shown in Figure 2 (e) indicates the distribution of noise and aliasing artifacts. The g-factor is generally greater than 1 , and the higher value of g-factor represents higher level of noise. For this reason, we set threshold of g-factor with different values to test the perceptron online learning algorithm. As indicated in the Table 2, 3 thresholds (1.0, 1.5, and 2.0) are list for performance comparison of the perceptron algorithm. Different thresholds represent different levels of noise. The 
Yuchou Chang; Real-Time Noise Classification of Medical Image via Online Machine Learning Algorithm. Advances in I mage and Video Processing, Volume 5 No 6, December (2017); pp: 1-9

higher value means only very noisy pixels are considered as noise in the image and the tolerance of noise is also higher. For the g-factor map, if a pixel value is greater than threshold, it is considered as the noisy pixel. Otherwise, it is considered as a pixel with true value under acceptable noise level. In addition, the reference MR brain image 2 is shown in Figure 3 (a). It is completely sampled but requires longer MR scanner acquisition time. Figure 3 (b) and (c) show a coil image and its coil sensitivity map. Coil sensitivity creates noise and aliasing artifacts in the reconstructed MR image as shown in Figure 3 (d). The corresponding geometric factor map as shown in Figure 3 (e) indicates the distribution of noise and aliasing artifacts in Figure $3(\mathrm{~d})$.

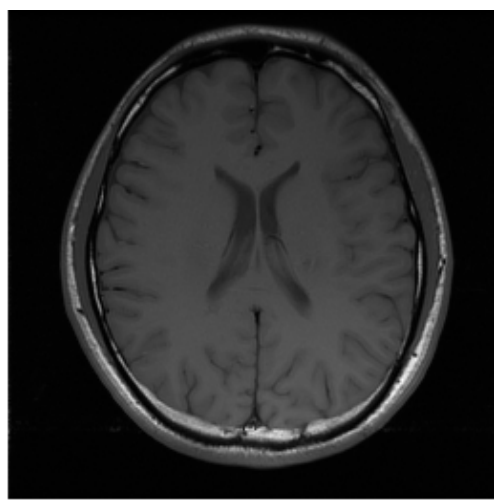

(a)

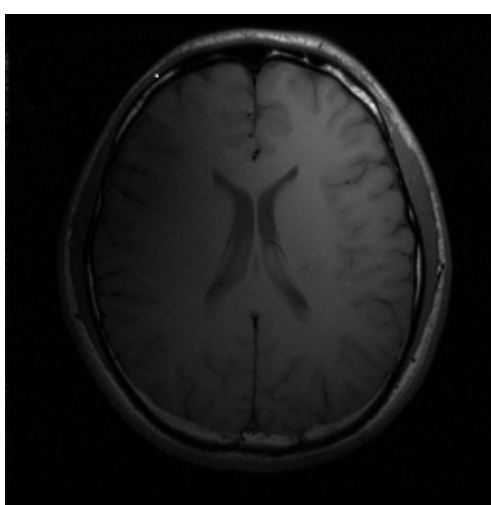

(b)

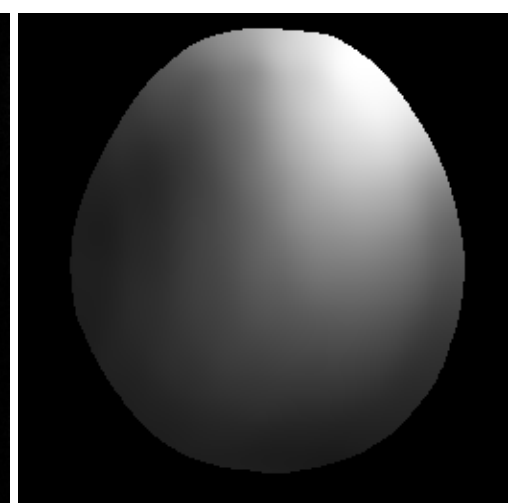

(c)

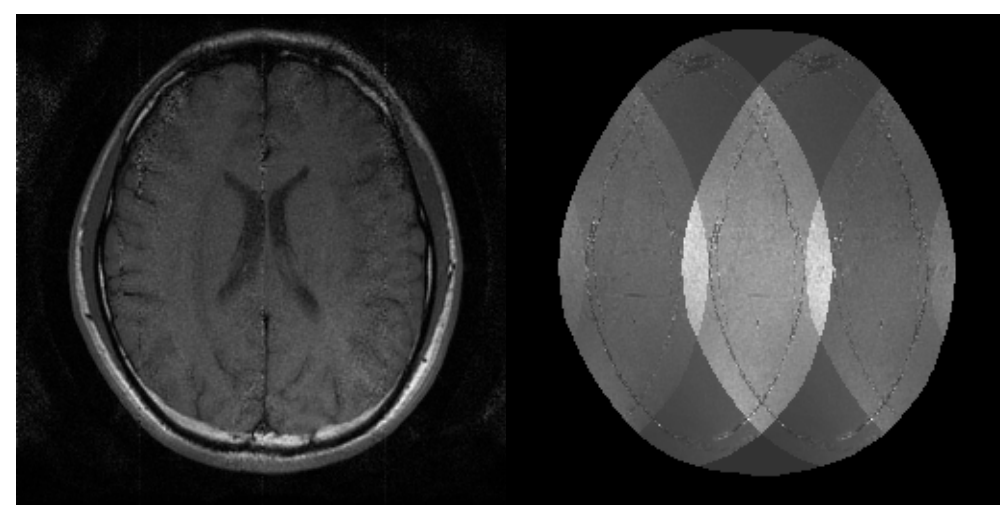

(d)

(e)

Figure 2. The original brain MR image $1(a)$, one coil image (b), the coil sensitivity map (c) related to the coil image (b), reconstructed image by SENSE (d), and g-factor map (e) with noise distribution in (d).

In addition, different numbers of pixel samples are randomly selected from 256 x 256 MR images each time for a trial. For the gray value of each pixel selected at each time, 8 neighbor pixel gray values are combined with this center pixel gray value are to obtain a feature vector to represent noisy level in the local $3 \times 3$ window. Feature vectors corresponding to the selected pixels are fed into perceptron classification algorithm in real-time. According to misclassification rate each time, classifier is dynamically adjusted based on the algorithm presented in the above section. For each time, the online cumulative mistake rates are list in Table 2. There are 30 times of trials in a sequence for the noise and non-noise pixels classification for both of brain images. Different brain image with different selection of threshold produces different cumulative mistake rate in real-time classification. For example, MR brain image 1 is T1 weighting, whose cumulative mistake rate is around 15\% along 30 times of trials when g-factor threshold is set as 1.0 , as indicated in the second column of the Table 2 . The mean value 
is $15.1 \%$ and the standard deviation is $0.2 \%$. Similarly, for other configurations of g-factor thresholds on both of brain image 1 and image2, each column shows changes of cumulative mistake rates of online classifications. The mean values are $26.6 \%$ (brain image 1 with g-factor threshold 1.5), $29.2 \%$ (brain image 1 with g-factor threshold 2.0), 21.2\% (brain image 2 with g-factor threshold 1.0), 38.5\% (brain image 2 with g-factor threshold 1.5), and 21.0\% (brain image 2 with g-factor threshold 2.0), respectively. The standard deviations values are $0.2 \%$ (brain image 1 with g-factor threshold 1.5 ), $0.2 \%$ (brain image 1 with g-factor threshold 2.0), 0.2\% (brain image 2 with g-factor threshold 1.0), 0.2\% (brain image 2 with g-factor threshold 1.5), and 0.2\% (brain image 2 with g-factor threshold 2.0), respectively.

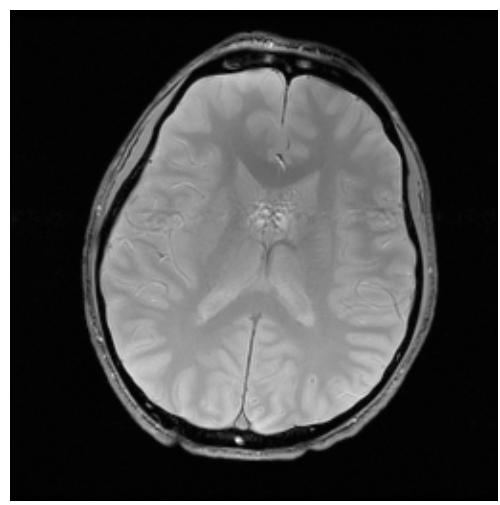

(a)

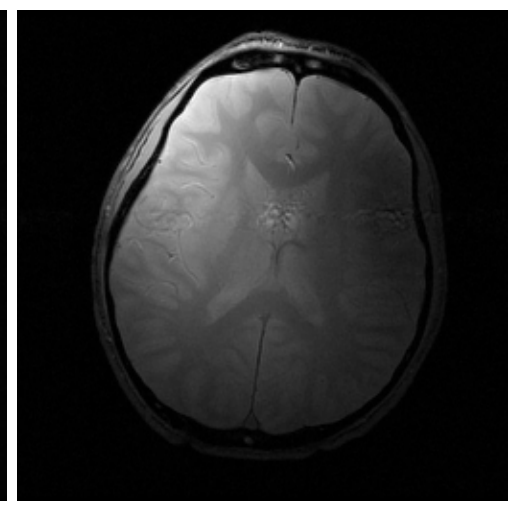

(b)

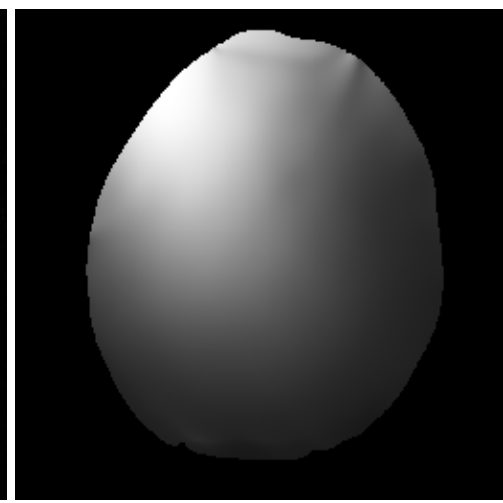

(c)

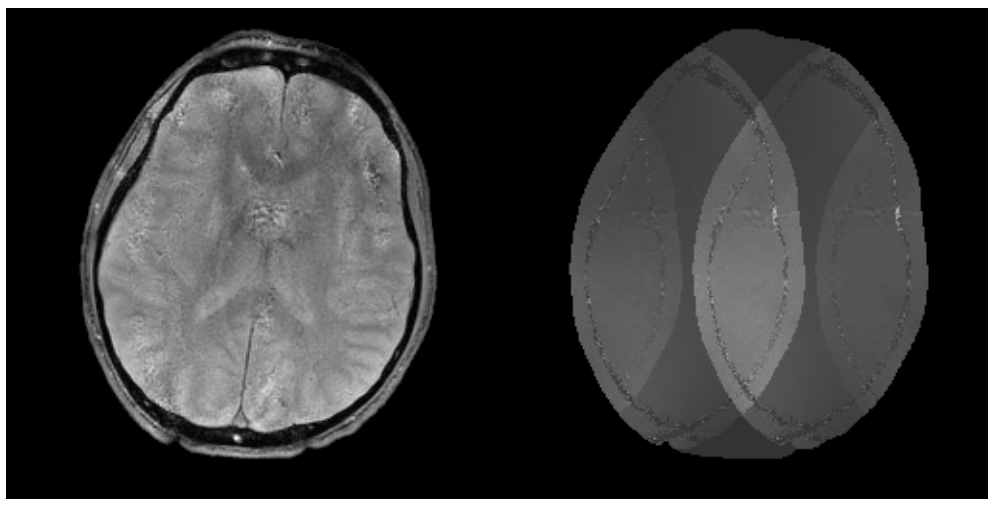

(d)

(e)

Figure 3. The original brain MR image 2 (a), one coil image (b), the coil sensitivity map (c) related to the coil image (b), reconstructed image by SENSE (d), and g-factor map (e) with noise distribution in (d).

Table 2. Classification of noisy pixels with different noisy levels for two brain MR images shown in the Figure 2 and Figure 3. Cumulative mistake rate is used as the measure.

\begin{tabular}{|c|c|c|c|c|c|c|}
\hline & Brain1, g1.0 & Brain1, g1.5 & Brain1, g2.0 & Brain2, g1.0 & Brain2, g1.5 & Brain2, g2.0 \\
\hline t-1 & $15.149 \%$ & $26.717 \%$ & $29.364 \%$ & $20.941 \%$ & $38.040 \%$ & $20.943 \%$ \\
\hline t-2 & $15.079 \%$ & $27.048 \%$ & $29.190 \%$ & $21.555 \%$ & $38.597 \%$ & $21.115 \%$ \\
\hline t-3 & $14.838 \%$ & $26.572 \%$ & $29.149 \%$ & $21.008 \%$ & $38.396 \%$ & $21.344 \%$ \\
\hline t-4 & $15.007 \%$ & $26.720 \%$ & $29.155 \%$ & $21.478 \%$ & $38.750 \%$ & $21.204 \%$ \\
\hline t-5 & $14.772 \%$ & $26.735 \%$ & $29.303 \%$ & $21.329 \%$ & $38.512 \%$ & $20.555 \%$ \\
\hline t-6 & $14.742 \%$ & $26.859 \%$ & $29.109 \%$ & $21.388 \%$ & $38.156 \%$ & $20.789 \%$ \\
\hline t-7 & $15.121 \%$ & $26.134 \%$ & $29.442 \%$ & $21.545 \%$ & $38.608 \%$ & $20.970 \%$ \\
\hline
\end{tabular}


Yuchou Chang; Real-Time Noise Classification of Medical Image via Online Machine Learning Algorithm. Advances in I mage and Video Processing, Volume 5 No 6, December (2017); pp: 1-9

\begin{tabular}{|c|c|c|c|c|c|c|}
\hline$t-8$ & $15.396 \%$ & $26.620 \%$ & $29.514 \%$ & $20.657 \%$ & $38.449 \%$ & $21.111 \%$ \\
\hline$t-9$ & $14.879 \%$ & $26.324 \%$ & $29.181 \%$ & $21.133 \%$ & $38.237 \%$ & $20.825 \%$ \\
\hline$t-10$ & $14.844 \%$ & $26.254 \%$ & $29.002 \%$ & $21.021 \%$ & $38.155 \%$ & $20.946 \%$ \\
\hline$t-11$ & $15.245 \%$ & $26.485 \%$ & $29.404 \%$ & $21.164 \%$ & $38.361 \%$ & $21.207 \%$ \\
\hline$t-12$ & $15.033 \%$ & $26.271 \%$ & $29.105 \%$ & $21.037 \%$ & $38.210 \%$ & $20.920 \%$ \\
\hline$t-13$ & $14.944 \%$ & $26.825 \%$ & $29.184 \%$ & $21.136 \%$ & $38.242 \%$ & $20.961 \%$ \\
\hline$t-14$ & $14.986 \%$ & $26.651 \%$ & $29.280 \%$ & $21.359 \%$ & $38.777 \%$ & $20.956 \%$ \\
\hline$t-15$ & $15.271 \%$ & $26.685 \%$ & $29.185 \%$ & $21.088 \%$ & $38.599 \%$ & $21.037 \%$ \\
\hline$t-16$ & $15.439 \%$ & $26.506 \%$ & $29.512 \%$ & $21.690 \%$ & $38.560 \%$ & $21.042 \%$ \\
\hline$t-17$ & $15.337 \%$ & $26.717 \%$ & $29.366 \%$ & $21.701 \%$ & $38.449 \%$ & $21.104 \%$ \\
\hline$t-18$ & $15.329 \%$ & $26.762 \%$ & $29.282 \%$ & $21.364 \%$ & $38.411 \%$ & $21.033 \%$ \\
\hline$t-19$ & $15.254 \%$ & $26.700 \%$ & $29.239 \%$ & $21.295 \%$ & $38.542 \%$ & $21.086 \%$ \\
\hline$t-20$ & $14.899 \%$ & $26.526 \%$ & $29.523 \%$ & $21.124 \%$ & $38.644 \%$ & $21.193 \%$ \\
\hline$t-21$ & $15.034 \%$ & $26.602 \%$ & $29.065 \%$ & $21.465 \%$ & $37.958 \%$ & $21.071 \%$ \\
\hline$t-22$ & $15.237 \%$ & $26.253 \%$ & $29.147 \%$ & $21.214 \%$ & $38.513 \%$ & $21.054 \%$ \\
\hline$t-23$ & $15.048 \%$ & $26.869 \%$ & $29.181 \%$ & $21.371 \%$ & $38.492 \%$ & $20.932 \%$ \\
\hline$t-24$ & $14.996 \%$ & $26.228 \%$ & $29.172 \%$ & $21.263 \%$ & $38.765 \%$ & $21.266 \%$ \\
\hline$t-25$ & $14.706 \%$ & $26.265 \%$ & $29.312 \%$ & $20.985 \%$ & $39.008 \%$ & $20.862 \%$ \\
\hline$t-26$ & $15.248 \%$ & $26.520 \%$ & $28.920 \%$ & $21.245 \%$ & $38.214 \%$ & $21.362 \%$ \\
\hline$t-27$ & $14.871 \%$ & $26.497 \%$ & $29.221 \%$ & $21.448 \%$ & $38.733 \%$ & $20.950 \%$ \\
\hline$t-28$ & $15.408 \%$ & $26.604 \%$ & $29.543 \%$ & $21.097 \%$ & $38.286 \%$ & $20.964 \%$ \\
\hline$t-29$ & $15.219 \%$ & $26.674 \%$ & $29.182 \%$ & $21.176 \%$ & $38.402 \%$ & $21.062 \%$ \\
\hline$t-30$ & $15.228 \%$ & $26.752 \%$ & $29.175 \%$ & $21.074 \%$ & $38.475 \%$ & $21.158 \%$ \\
\hline
\end{tabular}

\section{Conclusion}

An online learning algorithms is applied for classifying noisy pixels of MR brain images. The real-time perceptron algorithm enhances speed of noise classification in SENSE MR images, which have been widely used on clinical applications. Classifier parameters are dynamically updated based cumulative mistake rate produced each time during the classification procedure. In addition, real-time classification is able to provide quick noise information. For the future work, multi-class perceptron algorithms will be investigated to identify multiple levels of noises in the dynamic MR images.

\section{REFERENCES}

[1] Gondara, L, Medical image denoising using convolutional denoising autoencoders. Data Mining Workshops (ICDMW), IEEE 16th International Conference on 2016.

[2] Kohan, M.N., et al., Denoising medical images using calculus of variations. Journal of Medical Signals and Sensors, 2011. 1(3): p. 184-190.

[3] Seetha J., et al., Denoising of MRI images using filtering methods. Wireless Communications, Signal Processing and Networking (WiSPNET), International Conference on 2016. 
[4] Shen, D., Deep learning in medical image analysis. Annual Review of Biomedical Engineering, 2017. 19: p. 221-248.

[5] Zhou, S.k., Medical image recognition, segmentation and parsing: machine learning and multiple object approaches. The Elsevier and Miccai Society Book Series, Academic Press 2016.

[6] Guido, T., et al., Model attraction in medical image object recognition. Proceedings of the SPIE, 1995. 2436(1): p. 18-29.

[7] Olesen O.V., et al., Motion tracking for medical imaging: a nonvisible structured light tracking approach. Medical Imaging, IEEE Transactions on, 2012. 31(1): p. 79-87.

[8] Lim J.H., et al., Motion tracking in medical images. Biomedical Image Understanding, Methods and Applications, 2015.

[9] Huang C., et at., Real-time 3D motion tracking using MR micro-coils for PET imaging. The Journal of Nuclear Medicine, 2013. 54(2): 44.

[10] Cleary, K., et al., Image-guided interventions: technology review and clinical applications. Annual Review of Biomedical Engineering, 2010. 12: p. 119-142.

[11] Shalev-Shwartz, S., Online learning and online convex optimization. Foundations and Trends in Machine Learning, 2011. 4(12: p. 107-194.

[12] Hoi, S.C.H, LIBOL: a library for online learning algorithms. Journal of Machine Learning Research, 2014. 12(1): p. 495-499.

[13] Pruessmann, K.P., et al., SENSE: sensitivity encoding for fast MRI. Magnetic Resonance in Medicine, 1999. 45(2): p. 952-962.

[14] URL: http://www.mr-tip.com/serv1.php?type=cam.

[15] Rosenblatt, F., The perceptron: a probabilistic model for information storage and organization in the brain. Psychological Review, 1958. 65(6): p. 386-408.

[16] Cesa-Bianchi, N., et al. A second-order perceptron algorithm. SIAM Journal on Computing, 2005. 34(3): 640-668.

[17] Pieciak, T., et al., Non-stationary rician noise estimation in parallel MRI using a single image: a variance-stabilizing approach. Pattern Analysis and Machine Intelligence, IEEE Transactions on, 2017. 39(10): p. 2015-2029.

[18] Aja-Fernandez, S., et al., Statistical noise analysis in GRAPPA using a parametrized noncentral Chi approximation model. Magnetic Resonance in Medicine, 2011. 65(4): p. 1195-1206. 